\title{
The Time of Flight Upgrade for CLAS at 12GeV
}

\author{
Lewis Graham \\ Department of Physics and Astronomy \\ University of South Carolina \\ 712 Main Street \\ Columbia, SC 29208, USA
}

\begin{abstract}
The Time of Flight (TOF) system is a detection system within the CEBAF Large Acceptance Spectrometer (CLAS) at Jefferson National Accelerator Facility. CLAS, being a magnetic toroidal multi-gap spectrometer, is used in the detection of particles and their varying properties. Jefferson National Accelerator Facility is providing an incoming electron beam of energy $6 \mathrm{GeV}$ that is used to probe the structure and production of these particles. The CLAS detector is currently adapted to energies of up to $6 \mathrm{GeV}$, but with recent approval it will now upgrade to energies of $12 \mathrm{GeV}$. CLAS consists of drift chambers to determine the charged particle paths, gas Cherenkov counters for electron discrimination, TOF scintillators for particle identification, and an electromagnetic calorimeter for identifying showering electrons and photons. The TOF system, which is our focus, is composed of scintillation counters at the forward angle, and covers an area of 206 meters squared. Therefore, we look to upgrade and construct the TOF system of CLAS and outline strategies of current construction, purpose for design, and outlook for the TOF system upgrade
\end{abstract}

Keywords: CLAS; Time of Flight; Time Resolution.

PACS: 29.40.-n, 82.80.Rt, 29.40.Mc

\section{INTRODUCTION}

The Time of Flight (TOF) system is designed to decipher the flight time of particles produced from incident radiation reactions. If a particle's start position and momentum are known, then taking into account the information from where it was detected at the end of its flight time, one can calculate the flight time of that particular particle. The TOF system was purposed to achieve good timing resolution for identifying particles and good segmentation for flexible triggering and pre-scaling. It is divided into two subdivisions that are parameterized by the scattering angle of the reaction particle. At angles of $0^{\circ}$ to $40^{\circ}$, TOF is maximized by what are known as the small (forward) angle counters where the maximum counting rate in an electron beam experiment occurs and the average rate per scintillator is about $100 \mathrm{kHz}$. From $41^{\circ}$ and above, these are known as the large angle counters. The TOF system is equipped for a time resolution of $\sigma=120 \mathrm{ps}$ at the smallest angles and $250 \mathrm{ps}$ at angles of $90^{\circ}$ and up. These specifications are required by the fact that at small angles, the most energetic and thus fastest particles are produced. Therefore, the system is required to provide signals that represent a uniform response to properly identify particles that reach the time of flight detectors. In addition to excellent timing resolution and segmentation, the TOF system must be able to operate in a high-rate environment.

CP947, VII Latin American Symposium on Nuclear Physics and Applications

edited by R. Alarcon, P. L. Cole, C. Djalali, and F. Umeres

(C) 2007 American Institute of Physics 978-0-7354-0461-8/07/\$23.00 
Our overall goal is to improve the resolution by a factor of two, reaching $\sigma=60 \mathrm{ps}$ at forward angles. Given that all detectors must be optimized for best results, the time of flight system for CLAS is one of several detectors being upgraded to unveil and give greater opportunity to new and exciting physics. For this reason, we have taken on the challenge and lead in prototyping and eventually building the new time of flight system that will operate at $12 \mathrm{GeV}$.

\section{Materials}

To affectively achieve an overall resolution of $60 \mathrm{ps,} \mathrm{many} \mathrm{factors} \mathrm{contribute} \mathrm{in}$ meeting this goal. Our setup consists of a scintillation bar with (or without) light guides and photomultiplier tubes on each end that connect to the electronics for recording and processing their signals.

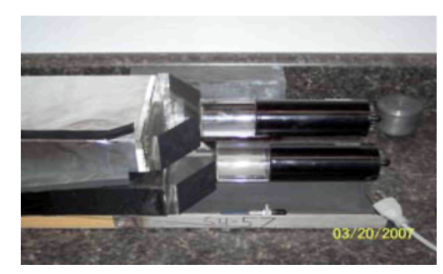

FIGURE 1. Scintillation bar with light guide and photomultiplier tube attached.

From a radioactive source on the scintillator, light is produced and propagates through the scintillation bar into the light guide where it is directed into the surface of the photomultiplier tube (PMT). The PMT, which is connected to high voltage and covered by magnetic shielding, converts the light signal into an electric current that is passed on to electronic modules to be eventually converted into digital information then recorded for analysis. The scintillation material is selected to optimize over the volume of the counter with a fast response. It must have a fast time response, low light attenuation, light-tight housing and it has to be transparent to emitted light. The photomultiplier tubes are used to cover a large area while maintaining good time resolution. They must process light with correct frequency spectrum as produced by the scintillator and also convert low light intensity into electrical signal. Voltage dividers use high-voltage field effect

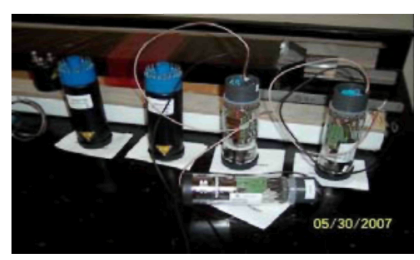

FIGURE 2. PMTs and voltage dividers used. 
transistors to fix the PMT gain by stabilizing the voltage and to protect the PMT against high light levels when it would shut down the circuit if there was an overcurrent. Light guides are used to connect the scintillators to the PMTs to optimize the light collection. They allow more uniform light collection from tracks traversing corners. Magnetic shielding is also used to cover the PMTs to reduce axial and traverse components of the magnetic stray field coming from the CLAS toroidal magnet.

\section{Procedure}

To measure the time of flight, you first have a particle passing through the scintillator bar from where an analog signal is sent to a discriminating electronic module.

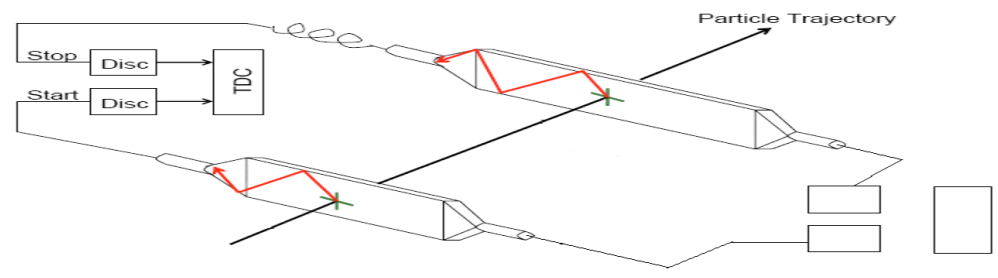

FIGURE 3. Schematic of particle passing through scintillator bars emitting light for signal response.

The discriminator converts the analog signal to a digital signal by accepting only values above a pre-selected threshold. This digital signal is then sent to a Time to Digital Converter (TDC) electronic module as a start time for data recording. After the particle has passed through the second scintillator, a stop time for data taking is sent. Time information is stored for both ends of the scintillator and later processed by computer to compensate for position dependent light transmission times. From the collection of data and timing information, we can measure an overall resolution. Where our electronics $\left(\sigma_{0}\right)$, scintillator and PMT $\left(\sigma_{1}\right)$, path-

$$
\sigma_{\mathrm{TOF}}(\mathrm{ns})=\sqrt{\sigma_{0}^{2}+\frac{\sigma_{1}^{2}+\left(\sigma_{\mathrm{P}} L / 2\right)^{2}}{N_{\mathrm{pe}} \exp (-L / 2 \lambda)}}
$$

FIGURE 4. Equation for time resolution. 
length variation $\left(\sigma_{\mathrm{p}}\right)$, and number of photoelectrons $\left(\mathrm{N}_{\mathrm{pe}}\right)$ all contribute to our final parameterization of the time resolution.

\section{Goals and Outlook}

The CLAS upgrade at $12 \mathrm{GeV}$ looks to accomplish extending particle identification to higher momenta, better charged particle traveling resolution, improved two pion separations, and reducing the width of scintillation bars from $15 \mathrm{~cm}$ to $5 \mathrm{~cm}$ to increase the number of photoelectrons for improved time resolution. Where CLAS currently has a pion-kaon separation up to $2 \mathrm{GeV}$, with a resolution of 60 ps we can improve this separation to $3 \mathrm{GeV}$ and a proton-pion separation of $6 \mathrm{GeV}$.

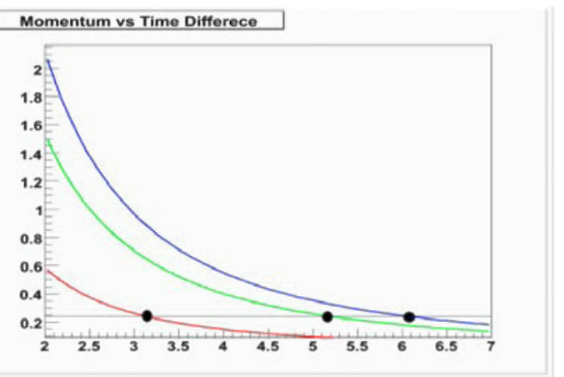

FIGURE 5. Plot shows time difference versus energy for pion-kaon (left), proton-kaon (middle), proton-pion (right)

It is our conclusion that with adequate electronic modules and optimized detector components such as PMTs and magnetic shielding, we will reach a time resolution of $60 \mathrm{ps}$ for the $12 \mathrm{GeV}$ upgrade at CLAS. We are currently performing various tests such as: discriminator testing of threshold dependencies and leading-edge versus constant fraction, TDC testing of integral linearity and differential non-linearity, cable attenuation testing, and finally magnetic mu-metal shielding testing. All are important tests in helping to achieve our overall time resolution. With the completion of these tests, we will ultimately finish the new detector design.

\section{ACKNOWLEDGMENTS}

I would like to thank R. Gothe, K. Park, H. Lu, Z. Zhao, E. Phelps, D. Gothe, L. Torres, and C. Eaker for their help, work and efforts in this project.

\section{REFERENCES}

1. E. S. Smith, The time-of-flight system for CLAS, Nuclear Instruments and Methods A 432, 265-298 (1999). 